\title{
Complicaciones orales en niños post-terapia antineoplásica
}

\author{
Oral complications in children after cancer therapy
}

Samantha Rivas Urbina ${ }^{1}$

Lorena Flores Barrantes ${ }^{2}$

Antonio Wachtel A. ${ }^{3}$

\section{Resumen}

Las neoplasias malignas han ido adquiriendo a lo largo de los últimos años una prevalencia significativa. Esta enfermedad no excluye a la población infantil, aunque se presenta en baja frecuencia, constituye una de las tres causas de muerte en niños.

El tratamiento antineoplásico a través de los años ha logrado porcentajes altos de curaciones, sin embargo, los grados de toxicidad que afectan a las células normales han aumentado por la intensidad de los tratamientos, siendo la cavidad oral muy susceptible a los efectos adversos directos e indirectos de la quimioterapia y radioterapia, debido a un alto índice de renovación celular. Dichas manifestaciones orales de carácter aguda o crónicas se ven agravadas por los problemas odontológicos preexistentes (caries, gingivitis y mala higiene).

Es importante para el odontólogo conocer dichas alteraciones, identificarlas y así poder asistir adecuadamente a los pacientes para mejorar la calidad de vida. En este artículo se describen las alteraciones que se presentan con más frecuencia así como la experiencia y manejo clínico dentro del servicio de odontopediatría del Instituto Nacional de Enfermedades Neoplásicas.

Palabras Claves: mucositis, cáncer infantil, prevención.

\begin{abstract}
Malignant tumors have acquired a significant prevalence over the past few years. This disease does not exclude the pediatric population, even though with a low frequency it is one of the three causes of death in children.
\end{abstract}

The antineoplastic treatment has improved the cure rate with high percentages of disease free survivors, but the degree of toxicity that affect normal cells has increased due to the intensity of treatments. The oral cavity is very susceptible to the direct and indirect adverse effects of chemo and radiotherapy, due to a high cell renovation index. These acute or chronic oral manifestations are aggravated with pre existent dental problems (cavities, gingivitis, bad hygiene).

It is important for the dental specialist to be aware of these abnormalities, identify them and thus treat the patients accordingly to improve

\footnotetext{
${ }^{1}$ Profesor de Odontopediatría Dpto. Pediatría Instituto Nacional de Enfermedades Neoplásicas Lima - Perú, Especialista en Odontopediatría Universidad Peruana Cayetano Heredia.

${ }^{2}$ Residente del programa de Especialización en Odontología Pediátrica. Facultad de Estomatología, Universidad Peruana Cayetano Heredia.

${ }^{3}$ Profesor Asociado, Dpto. Pediatría, Universidad Peruana Cayetano Heredia, Doctor en Medicina, Médico Asistente Dpto. Pediatría Instituto Nacional Enfermedades Neoplásicas Lima, Perú.
} 
their quality of life. In the present paper we describe the most common abnormalities encountered as well as the experience and management in the Pediatric Dental Service at the Instituto Nacional de Enfermedades Neoplasicas.

Key words: mucositis, childhood cancer, prevention

\section{Introducción}

El cáncer infantil se presenta en una frecuencia baja, tanto entre las enfermedades pediátricas, como entre las enfermedades neoplásicas de la población en general, pero constituye una de las tres causas más frecuentes de muerte en niños mayores de un año, seguida por los accidentes infantiles ${ }^{1,2,3,4}$. La incidencia anual oscila en el mundo entre 120 a 150 casos nuevos por millón de personas menores de 15 años, variando según la edad, sexo, raza y localización geográfica. En el Instituto Nacional de Enfermedades Neoplásicas (INEN) se atienden anualmente un promedio de 635 casos nuevos en el área de pediatría. En las últimas décadas se ha observado tanto un aumento de la incidencia como una disminución global en su morbilidad, debido a los importantes avances diagnósticos y terapéuticos $^{1,5}$.

De todas las neoplasias infantiles, las más frecuentes son las leucemias en sus distintas presentaciones, que constituyen el tercio del total (entre ellas, más del 80\% son leucemias linfoblásticas agudas) y cerca de la mitad de todos los cánceres en la infancia, si se considera conjuntamente las leucemias y los linfomas. El resto de las neoplasias infantiles son el neuroblastoma $(8 \%)$, tumor de Wilms $(7 \%)$, sarcomas de partes blandas $(6 \%)$, tumores óseos $(5 \%)$, retinoblastomas $(8 \%)$, hepatoblastomas (2-3\%) o tumores de células germinales, que aún siendo menos fre- cuentes son característicos, porque prácticamente sólo se encuentran en la infancia ${ }^{1,4,5,6,7,7,8}$.

El diagnóstico precoz va a ser decisivo en cuanto al pronóstico. Sin embargo, el tratamiento antineoplásico puede producir efectos tóxicos inevitables en las células normales. El recubrimiento mucoso del tracto digestivo, incluyendo la mucosa bucal, es el blanco principal de los efectos secundarios relacionados con el tratamiento a causa de su alta tasa de proliferación celular. Es así, que los cambios en la estructura de los tejidos blandos de la cavidad oral se van a ver reflejados en todo el tracto gastrointestinal ${ }^{2,45,6,6,8}$.

Los efectos tóxicos en la cavidad oral, secundarios a los tratamientos requieren de programas odontológicos preventivos para evitar y tratar en forma temprana las lesiones. Estas complicaciones orales ocurren en la mayoría de los pacientes tratados con quimioterapia y radioterapia. En mayor porcentaje se presentan en aquellos pacientes con neoplasias a nivel de cabeza y cuello. $28,9,10,1,1,12,13$

Entre los tratamientos antineoplásicos usados en niños tenemos la quimioterapia y radioterapia según el caso, así como tratamientos quirúrgicos complementarios. En la actualidad la radioterapia para el sistema nervioso central en leucemias linfáticas ha sido sustituida por la quimioterapia de tipo intratecal, sin embargo, para los casos con más de 100,000 leucocitos al diagnóstico se recomienda el uso de radioterapia profiláctica. Debido a que la radioterapia, sobre todo a nivel de cabeza y cuello, va a provocar no sólo cambios específicos histológicos y fisiológicos de la mucosa oral, sino que también puede dar lugar a alteraciones estructurales y funcionales de los tejidos subyacentes de apoyo, incluyendo a las glándulas salivales, huesos y dientes. A nivel dental, si la irradiación fue generada al momento de la odontogénesis, va 
a haber una alteración en la replicación celular normal, y se producirán problemas de agenesia dental, anomalías de tipo, tamaño y forma, así como dificultad en la erupción 2,10,11,12,13.

Las complicaciones orales más frecuentes después de los tratamientos antineoplásicos, son la estomatitis o también llamada mucositis, infecciones orales por Herpes, Cándida o Pseudomona, hemorragias, xerostomía, y odontalgias, las cuales van a provocar a su vez efectos secundarios como deshidratación y desnutrición como consecuencia a la dificultad de ingerir y deglutir alimentos $2,3,6,9,10,14,15,16,17,18$.

\section{Estomatitis o mucositis}

Los términos mucositis y estomatitis se emplean alternativamente en la literatura sobre el cáncer para referirse a la toxicidad en los tejidos bucales como resultado de la quimioterapia o radioterapia ${ }^{9}$. La mucositis se define como la inflamación de las membranas de la mucosa a nivel de todo el tracto digestivo. La estomatitis, término mas especifico, se refiere a la inflamación de las membranas de la mucosa bucal $8,9,11,15,19,21,22$.

Los factores predisponentes de la estomatitis $\operatorname{son}^{3,6,13,15,19,20,21 \text { : }}$

- Pobre o nula higiene bucal.

- Nutrición deficiente.

- Terapia con antibiótico.

- Granulocitopenia.

- Tratamiento con esteroides.

La mucositis presenta una prevalencia que oscila entre 30 y $70 \%$ tras la administración de quimioterapia, pudiendo aumentar hasta un $90 \%$ en caso de trasplante de médula ósea ${ }^{15}$. Esto sucede debido a que la quimioterapia citotóxica afecta a las células que se encuentran en mayor actividad mitótica y en general, en pacientes jóvenes, quienes van a presentar gran actividad celular a nivel de las mucosas, son los que van a experimentar mayor incidencia de complicaciones $^{3,10,14,19}$. Los agentes antineoplásicos que van a causar con mayor facilidad daño en las mucosas son bleomicina, dactinomicina, doxorrubicina, entre otros. Otros factores causales además de la droga en sí, es el tipo de neoplasia, edad, conteo de neutrófilos e higiene y cuidado oral2,19. La mucositis se va a observar como una inflamación y ulceración de la mucosa, frecuentemente dolorosa, que aparece de 7 a 10 días después del inicio de la quimioterapia, y con una duración de varios días. La primera señal va a ser el eritema en el paladar blando, mucosa yugal, vientre de la lengua y/o piso de boca, seguido de edema, ulceración con posible sangrado y/o exuda$\mathrm{do}^{14,15,21}$. Algunos autores la separan en dos tipos, la eritematosa que ocurre en algunos casos antes de los 3 días, y la ulcerosa, que aparece a los siete días de iniciado el tratamiento y es la más grave y dolorosa. Los pacientes con mucositis suelen presentar sequedad en la boca, dificultad a la deglución, quemazón, hormigueo en los labios, dolor. Es por eso que afecta considerablemente la calidad de vida del paciente ${ }^{14,15,19,21}$.

Como consecuencia, existen posibilidades de infecciones secundarias y oportunistas, principalmente por Cándida albicans, con serio compromiso de la condición nutricional del paciente, que puede llegar a anorexia provocando deshidratación y desnutrición 4,16,17,18. La mucositis es una causa importante de morbilidad durante la terapia antineoplásica ${ }^{15}$.

La clasificación de los diferentes grados de mucositis se resume en la Tabla I. 
Tabla I. Clasificación según Hoogstraten²3.

\begin{tabular}{|c|c|}
\hline GRADOS & SINTOMAS \\
\hline 0 & Sin cambios aparentes. \\
\hline 1 & Irritación, eritema, se alimenta adecuadamente. \\
\hline 2 & Eritema, ulceraciones, puede comer alimentos sólidos con algo de dificultad. \\
\hline 3 & $\begin{array}{r}\text { Dolor en encías, edematosas, ulceraciones de la mucosa oral, alimentación líquida ex- } \\
\text { clusivamente. }\end{array}$ \\
\hline 4 & $\begin{array}{r}\text { Edema, eritema, úlceras en toda la cavidad oral, dolor para pasar la saliva, no puede } \\
\text { alimentarse ni tomar líquidos por dolor. }\end{array}$ \\
\hline
\end{tabular}

Sabater y col. ${ }^{11}$ describieron los factores de riesgo asociados a la aparición de mucositis. Entre ellos, las relacionadas con la pauta de quimioterapia y los relacionados con el propio individuo (edad, estado nutricional, estado buco-dental, estado hematológico, entre otros). Por otro lado, se ha sugerido que la mucositis no solo afectaría al epitelio, sino que algunos fenómenos celulares y moleculares se alcalizarían en la submucosa, principalmente en el endotelio vascular, así también existe alguna base genética en la aparición y gravedad de la mucositis ${ }^{9,10,15}$.

\section{Infecciones}

La mucositis oral puede complicarse con infecciones en los pacientes por inmunidad reducida, la pérdida del epitelio oral como barrera protectora da como resultado infecciones locales, proporciona una puerta de entrada para microorganismos a la circulación sistémica. Una vez que se vea afectada la integridad de la mucosa, las infecciones sistémicas y locales pueden ser causadas por la flora oral propia, así como también por organismos nosocomiales y oportunistas. Al reducirse la cantidad absoluta de neutrófilos por debajo de 1,000 por milímetro cúbico, la incidencia y gravedad de las infecciones se eleva. Los pacientes con neutropenia prolongada corren un riesgo mayor de desarrollar complicaciones infecciosas graves ${ }^{2,3,4,6,13,14,21}$.

La etiología de las úlceras es variada, incluyendo neutropenia, citotoxicidad a las drogas, infecciones fúngicas, bacterianas y virales, como también lesiones aftosas o eritema multiforme. Muchas ulceraciones ocurren después de las mucositis, pero en otras ocasiones, estas se desarrollan en forma independiente ${ }^{6,9,18,21}$. Dentro de los tipos de infecciones, tenemos a las de tipo viral, fúngicas y bacterianas ${ }^{4}$.

En pacientes inmunosuprimidos, la recurrencia intraoral puede presentarse en cualquier lugar de la boca, transformándose en lesiones severas que pueden complicarse y diseminarse con una morbilidad significativa y evolución más larga de lo normal, por lo que es de vital importancia prevenir la reactivación o detectarla precozmente. Los criterios clínicos tradicionales para distinguir lesiones bucales producidas por el virus herpes simple (VHS) de otras producidas por otros virus o lesiones no virales, no son aplicables en pacientes 
inmunosuprimidos, debido a la presentación clínica atípica que presentan estos pacientes ${ }^{5,18}$. Existen estudios que indican que la mayoría de las ulceraciones en pacientes inmunosuprimidos corresponden a lesiones causadas por VHS. Así como también se ha reportado, la reactivación del VHS en pacientes que reciben quimioterapia ${ }^{5,13,16,18,21}$. Además, en ellos se puede asociar a otros virus como citomegalovirus, o Epstein Barr ${ }^{5,18}$.

En cuanto a las lesiones fúngicas, la más frecuente es la candidiasis, que puede ocurrir en la forma pseudomembranosa caracterizada con placas, o en las formas eritematosa, atrófica crónica y queilitis angular ${ }^{4}$. La candidiasis oral es una complicación infecciosa aguda común en niños que reciben tratamiento de cáncer, sobretodo en periodos de neutropenia severa ${ }^{17}$. Existen diversos factores locales que van a provocar una infección por Cándida en la cavidad bucal, como una saliva ácida, xerostomía, uso nocturno de prótesis dentales, dieta rica en carbohidratos y pacientes que reciben radioterapia sobre tejidos de cabeza y cuello o quimioterapia. La colonización de especies de Cándida en pacientes con cáncer y su diseminación tardía, está asociada con episodios largos de neutropenia, uso de antibióticos de amplio espectro, tratamiento con corticoesteroides y sustancias citostáticas, procedimientos quirúrgicos invasivos, xerostomía y largo tiempo de hospitalización. La mortalidad por septicemia fúngica, asociada a infección primaria bucal, es un problema importante en niños con cáncer. Las especies de cándida, existentes en la boca, tienen una alta probabilidad de infectar la vía digestiva y así diseminarse por vía sanguínea, provocando una infección sistémica ${ }^{16,17,18}$.

\section{Hemorragia}

La hemorragia puede ocurrir durante episodios de trombocitopenia y/o coagulopatía inducida por el tratamiento 2 . Pueden aparecer lesiones purpúricas en la mucosa bucal, semejantes a equimosis cutáneas. Los sitios de enfermedad periodontal subyacente pueden sangrar espontáneamente o por un trauma mínimo. El sangrado oral puede ser leve, con petequias localizadas en los labios, el paladar blando o el piso de la boca, o puede ser grave presentándose con hemorragia oral, especialmente en los surcos gingivales ${ }^{2,4,6}$.

Cada intervención en los pacientes bajo quimioterapia debe ser analizada individualmente considerándose el estado general del paciente, el tipo y dosis de medicación administrada, pero como guía general para realizar cualquier procedimiento quirúrgico odontológico, los valores hematológicos deber ser, leucocitos por encima de $3000 / \mathrm{mm}^{3}$, neutrófilos $1500 / \mathrm{mm}^{3}$ y plaquetas por encima de $100000 / \mathrm{mm}^{32,4}$.

\section{Xerostomía}

La xerostomía o disminución de flujo salival es una de las complicaciones más frecuentes en la mayoría de los tratamientos antineoplásicos, debido a que la acción de las drogas altera temporalmente el mecanismo cuantitativo y cualitativo de la saliva, reduciendo la amilasa salival e Ig A, aumentando su viscosidad. Como consecuencia los pacientes van a presentar sensación de ardor y dolor en la boca, dificultad de deglutir los alimentos secos, dificultad de hablar, disminución del gusto. ${ }^{2,4,16}$.

Cuando está asociada a mucositis, la xerostomía puede llevar a ulceraciones, intensificando el dolor, además de favorecer las infecciones oportunistas $^{4,13,21}$.

Se presenta comúnmente en pacientes sometidos a terapias de radiación a nivel de cabeza y 
cuello. El efecto va a variar de paciente a paciente y va a depender de la cantidad de radiación y localización. Las glándulas salivales en el campo irradiado por lo general experimentan cambios funcionales irreversibles severos. La saliva puede tornarse espesa y viscosa, o se puede reducir el flujo severamente. Los elementos serosos de las glándulas salivales son mas radiosensibles, y los pacientes que reciben radiación a nivel de cabeza y cuello, regularmente pierden la mayor parte del componente seroso de la saliva. Cuando se afectan las glándulas mucosas, el paciente, puede perder la función de la glándula salival casi por completo, lo que provoca rápida y progresivamente caries inducida por radiación ${ }^{4,10}$, como consecuencia de la reducción de la saliva, secundaria a la terapia de radiación que disminuye uno de los protectores orales naturales en términos de capacidad Buffer y remineralización de las superficies dentales ${ }^{2,10}$.

La xerostomía va a causar diversos cambios en la boca, entre los cuales tenemos a la alteración salival, ésta se torna espesa y forma hilos, lo cual disminuye la lubricación siendo esto algo molesto para el paciente; se elimina su capacidad neutralizadora, en una boca muy limpia y seca, el $\mathrm{pH}$ es generalmente 4.5 pudiendo provocarse el proceso de desmineralización dental; la flora oral se hace más patógena; la placa se hace más espera y pesada, y los desechos permanecen en la boca debido a la incapacidad del paciente para limpiársela; no se depositan minerales (calcio, fósforo, fluoruro) en los dientes; y la producción de ácido después de la exposición al azúcar resulta en una ulterior desmineralización de los diente que provoca caries. ${ }^{2}$

\section{Consecuencias de la terapia de radiación}

El objetivo de la radiación es tratar el tejido tumoral impidiendo el crecimiento y reproducción celular provocando por último la muerte de la misma, mientras se restringe el daño a los tejidos circundantes9. La radiación local a la región de cabeza y cuello no sólo puede causar cambios específicos histológicos y fisiológicos de la mucosa oral provocados por la terapia citotóxica, sino también afectar a los tejidos subyacentes de apoyo, incluyendo a las glándulas salivales y los huesos ${ }^{2,9,10}$.

El odontólogo debe de considerar varios aspectos importantes del tratamiento. Las fuentes potenciales de infección del hueso radiado deben ser tratadas o eliminadas antes de iniciar la radioterapia $^{9,10}$.

La dosis elevada de radiación a los huesos que sostienen dientes causa reducción del suministro vascular a los huesos, y desintegración del tejido que conduce a la exposición del hueso y la necrosis ${ }^{2}$. La osteorradionecrosis es un efecto secundario a la terapia de radiación. La radiación ionizante origina que los canales vasculares se estrechen dentro de los campos irradiados, lo cual restringe el flujo sanguíneo y reduce la osteovascularidad. Estos efectos dejan la región con disminuida capacidad para reparar o defenderse en caso de trauma o infección. Mientras más radiación se reciba, mayor es el riesgo de osteorradionecrosis. Pacientes con osteorradionecrosis clínicamente pueden presentar dolor, trismus, supuración y ocasionalmente heridas de olor desagradable. Las glándulas salivales en el campo de la radiación por lo general experimentan cambios funcionales irreversibles y severos. La saliva suele tornarse espesa y viscosa, o puede reducir el flujo severamente, provocando una xerostomía severa ${ }^{9,10}$.

Niños bajo terapia de radiación pueden experimentar alteraciones significativas del crecimiento y desarrollo dental y de las estructuras 
faciales. Estas anormalidades incluyen a las raíces cortas, calcificación incompleta, desarrollo dental retrasado, hipodoncia, microdoncia, asimetría facial y relaciones oclusales alteradas ${ }^{10}$.

\section{Alternativas de tratamiento y manejo odontoestomatológico}

Se deberá efectuar en los distintos intervalos de tratamiento médico y posterior al mismo, lo más pronto posible. Consistirá en un primer momento en la evaluación odontológica completa y evaluaciones periódicas en la etapa de hospitalización. Durante esta etapa no se realizarán tratamientos odontológicos invasivos incluyendo el uso de cepillo dental, se optará por utilizar gasa con agua o pasta dental según la edad del paciente.

Existen controversias y distintos protocolos para el cuidado oral. La técnica Bass de cepillado y los enjuagues con agua salina bicarbonatada $u$ otras soluciones se recomiendan en pacientes bajo terapia oncológica siempre que los valores hematológicos lo permitan, ya que se puede generar sangrado gingival al utilizar cepillos dentales bajo cualquier técnica y provocar una hemorragia severa. Si el paciente presenta valores hematológicos inadecuados deberá limpiar la boca con ayuda de una gasa generando movimiento circulares suaves acompañado de algún enjuagatorio $^{19,23}$.

En las diferentes etapas del tratamiento médico el paciente puede tener distintos problemas en cavidad oral ya antes mencionados, que se producen por las terapias antineoplásica, es deber del odontólogo disminuir, aliviar dichas molestias y brindar mayor comodidad al paciente.

El uso de agentes analgésicos tópicos, geles bioadherentes para acondicionar la mucosa, uso de agentes de clorhexidina y terapias alternativas como el aloe vera, son de vital importancia para poder mejorar las condiciones de la cavidad oral.

El programa preventivo debe contar también con la aplicación de barnices fluorados, enjuagues con flúor durante la etapa de hospitalización. El uso de lubricantes labiales es de gran ayuda para prevenir la resequedad de los labios.

En caso de estomatitis o mucositis se propone dos tipos de terapias, tabla 2, un protocolo preventivo que deberá iniciarse al tercer día de quimioterapia para evitar aparición y/o aminorar su agresividad. El protocolo terapéutico, cuando las lesiones ya se encuentran instaladas.

Tabla 2. Protocolo de clorhexidina en el Departamento de Pediatría-INEN.

\begin{tabular}{|c|l|}
\hline $\begin{array}{c}\text { Protocolo } \\
\text { Preventivo }\end{array}$ & $\begin{array}{l}\text { Realizar higiene oral con gasa y agua o cepillo y pasta dental. } \\
\text { Colutorios o gel de clorhexidina al } 0.12 \% \text { sin alcohol una vez al día por } 7 \text { días. }\end{array}$ \\
\hline \multirow{2}{*}{$\begin{array}{c}\text { Protocolo } \\
\text { Terapéutico }\end{array}$} & $\begin{array}{l}\text { Pacientes mayores de } 6 \text { años: } \\
\text { Realizar higiene oral con gasa y agua o cepillo y pasta dental. } \\
\text { Uso de colutorios o gel de clorhexidina al } 0.12 \% \text { sin alcohol dos veces al día por } 15 \text { días. }\end{array}$ \\
\cline { 2 - 2 } & $\begin{array}{l}\text { Pacientes menores de } 6 \text { años: } \\
\text { Realizar higiene oral con gasa y agua o cepillo y pasta dental. } \\
\text { Uso de gel o spray de clorhexidina al } 0.12 . \% \text { sin alcohol dos veces al día por } 10 \text { días. }\end{array}$ \\
\hline
\end{tabular}


En ambos casos es importante una higiene oral previa con técnica no invasiva (gasa, agua o pasta dental) o con cepillo dental ultrasuave, esto dependerá del estado del paciente.

La clorhexidina es un antiséptico con amplio espectro antimicrobiano, bacteriostático con especial actividad sobre gram positivos, esporostático y fungistático. Su acción es rápida, presentando además una considerable persistencia y adherencia residual. Por sus características catiónicas poseen la propiedad de unión a la mucosa oral ${ }^{14,19}$.

El uso de clorhexidina en casos de mucositis oral ha sido ampliamente estudiado, presentando diversos resultados ${ }^{12,19}$. La clorhexidina ha demostrado que reduce las bacterias orales así como colonización fúngica. Es por eso que estos estudios recomiendan su uso como profilaxis en pacientes que van a recibir terapia no supresora ${ }^{19}$.

Otros agentes utilizados para casos de mucositis, es el gel oral bioadherente, cuyos componentes son la polivinilpirrovidona (PVP), el ácido hialúronico y ácido glicirrético el cual tiene propiedades antiinflamatorias. Este gel se aplica en la mucosa oral dejando una película delgada lo que permite al paciente ingerir alimentos, así como para reducción de sintomatología oral que acompaña a la mucositis ${ }^{11}$.

Este protector de la mucosa está indicado en diversas alteraciones de la mucosa oral, no sólo en el tratamiento de la mucositis, se utiliza para tratar otras lesiones inflamatorias orales ya que mejora la sintomatología inflamatoria, irritativa y traumática del área orofaríngea ${ }^{11,24}$.

Así mismo tenemos agentes naturales como es el aloe vera mas conocido como sábila, su conocimiento data de hace mucho tiempo. Su utilización como planta medicinal fue muy importante para las antiguas culturas como los griegos, romanos, egipcios, hebreos, asirios, árabes y para las culturas africanas, de donde se originaría ${ }^{24,25}$.

En la actualidad, y por sus características como regenerador celular y otras particularidades medicinales, se usan numerosas especies de aloe alrededor del mundo para condiciones que van desde dermatitis hasta cáncer. Se utiliza como ungüento superficial para quemaduras menores, abrasiones, heridas, ulceras malignas y otras lesiones epiteliales ${ }^{24}$.

Sus aplicaciones clinicas van desde pacientes con problemas de estreñimiento, acné vulgar, psoriasis vulgaris, hasta alteraciones de tipo dermatológicas como dermatitis seborreica, por radiación, entre otras. Pero su aplicación está también dirigida a la prevención de infecciones microbianas y mucositis ${ }^{24,25}$.

Es importante contar y conocer diferentes alternativas de tratamiento para brindarle al paciente diferentes soluciones.

\section{Experiencia clínica}

Concluyendo con nuestro artículo queremos brindarles algunas experiencias clínicas, que nos dejan ver sin duda la importancia del tratamiento multidisciplinario, para una satisfactoria calidad de vida.

Caso Clínico 1: Paciente de sexo femenino de 13 años de edad con diagnóstico Leucemia Linfática Aguda, quien cursaba con neutropenia severa. Al examen intraoral presentaba micosis en zona del paladar duro, y cultivo faríngeo positivo a pseudomona aeuriginosa. Se observa leve depresión en zona del paladar duro la cual se perfora en pocos días dejando comunicación buconasal, que ha persistido hasta su recuperación 


\section{Caso Clínico 1}

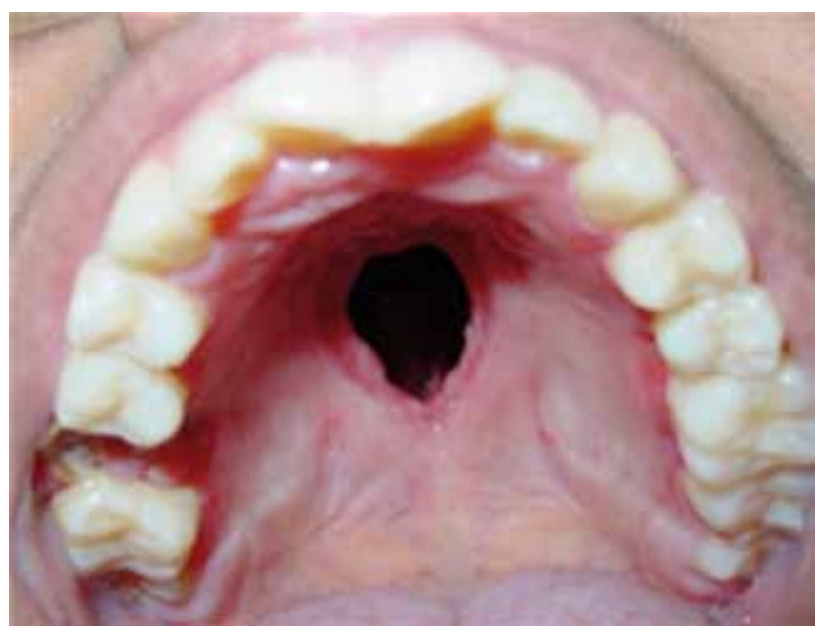

Figura 1. Vista de maxilar superior con comunicación buconasal.

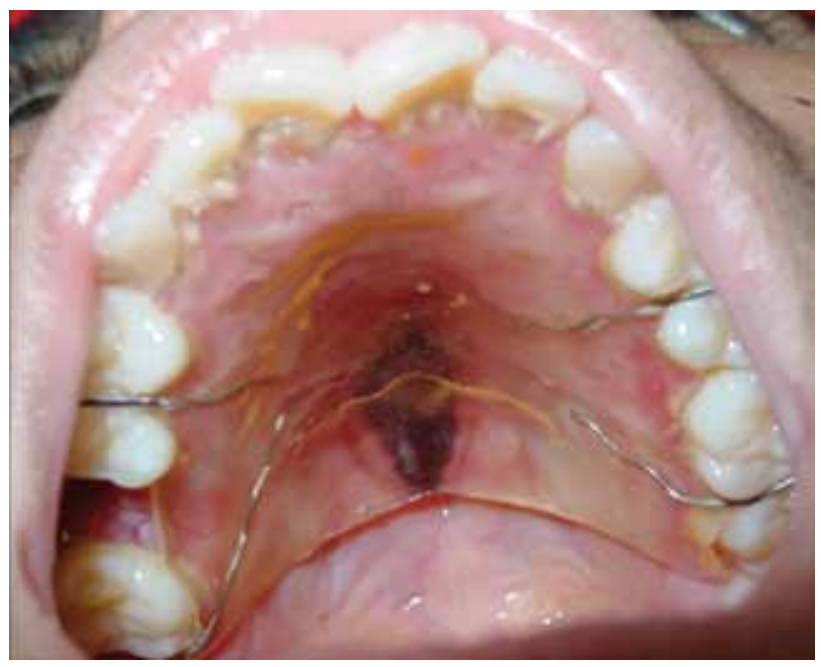

Figura 2. Vista de maxilar superior con obturador palatino.

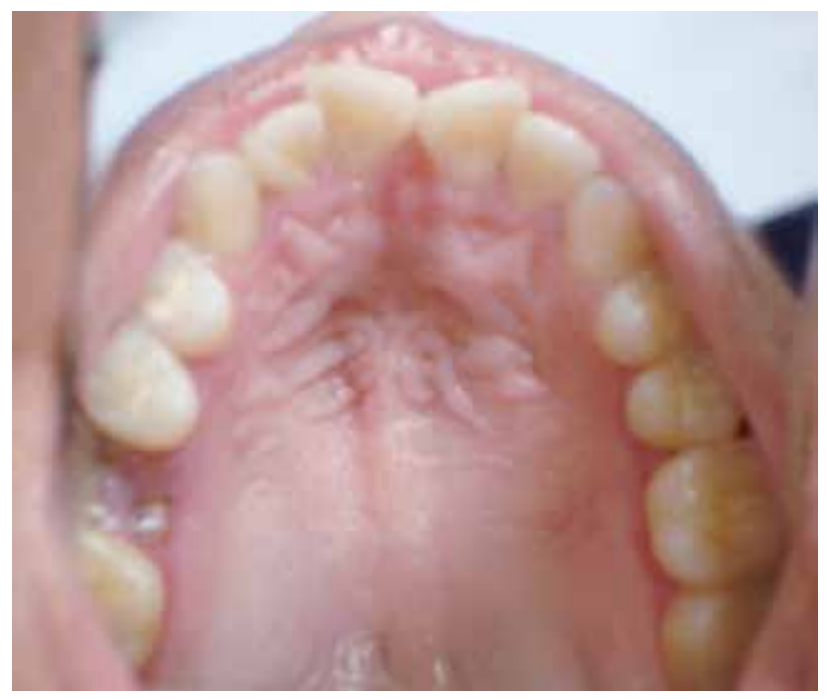

Figura 3. Vista de maxilar superior post-cirugía plástica. hematológica por lo que fue necesario para su alimentación, fonación adecuada, la colocación de una placa palatina u obturador, hasta poder llegar a la cirugía plástica reconstructiva del defecto óseo una vez concluido el tratamiento de la leucemia.

Caso Clínico 2: Paciente de sexo masculino de 13 años de edad, con antecedente de rabdomiosarcoma en seno maxilar a los 3 años de edad, el cual recibió tratamiento con quimioterapia y radioterapia. Termina tratamiento sin tener recidiva de la enfermedad. Después de 10 años llega a sus controles médicos y manifiesta presentar movilidad de algunas piezas dentarias. Por lo que es derivado al servicio de Odontopediatría. Al realizar el examen clínico estomatológico se observa depresión de la mejilla derecha, asimetría facial, línea de sonrisa alterada, gingivitis, abundante placa bacteriana, movilidad dentaria tipo 3 del sector anterior superior, no existe oclusión adecuada. Por lo que se indica radiografía panorámica en la que se evidencia una alteración en la morfología y estructura ósea del hemimaxiar superior derecho, con falta de desarrollo del su proceso alveolar, hipoplasia del seno maxilar ipsilateral y patrón trabecular atípico. En el maxilar inferior en la región posterior del lado derecho se obserba alteración, no se evidencia en forma completa la línea oblicua externa y las corticales del conducto dentario además de rarefacción ósea circundante a las piezas 46 y 47.

En la parte dentaria se evidencia hipoplasia radicular de piezas 22, 26 y 46; y aplasia radicular en las piezas 17, 13, 12, 11, 21 y 47. El germen dentario 27 evidencia aplasia radicular e hipoplasia coronaria con el cierre prematuro de sus estructuras. Además no se observa la cortical de su cripta ósea lo que sugiere inclusión en tejido blando; probablemente la fibrosis post radioterapia. 


\section{Caso Clínico 2}

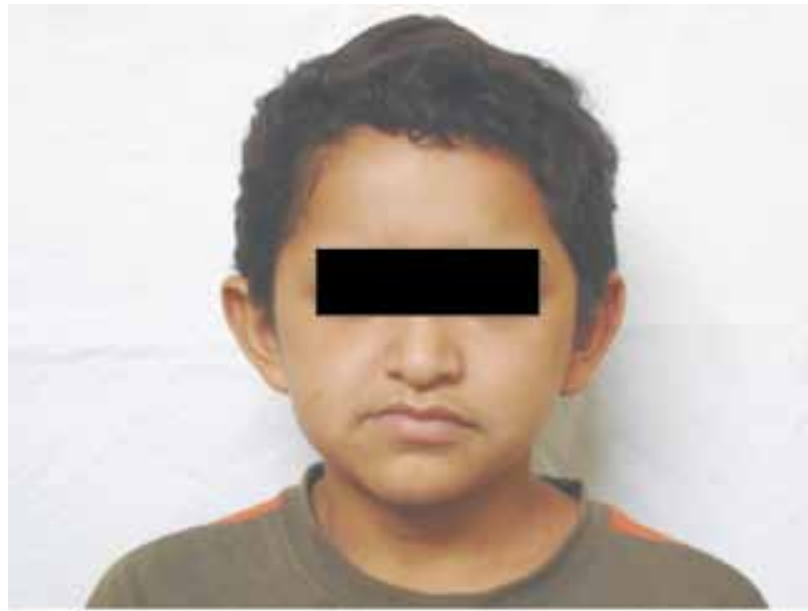

Figura 4. Vista frontal.

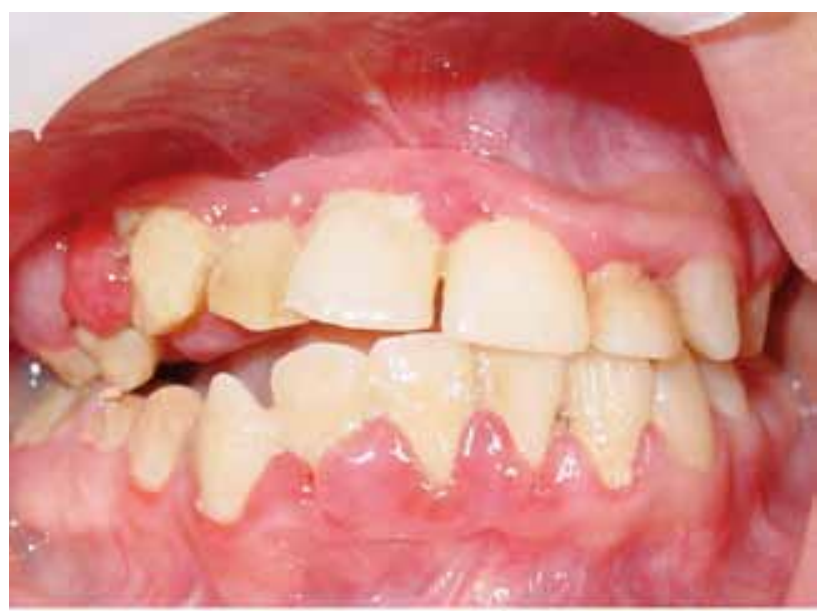

Figura 6. Vista intraoral inicial.

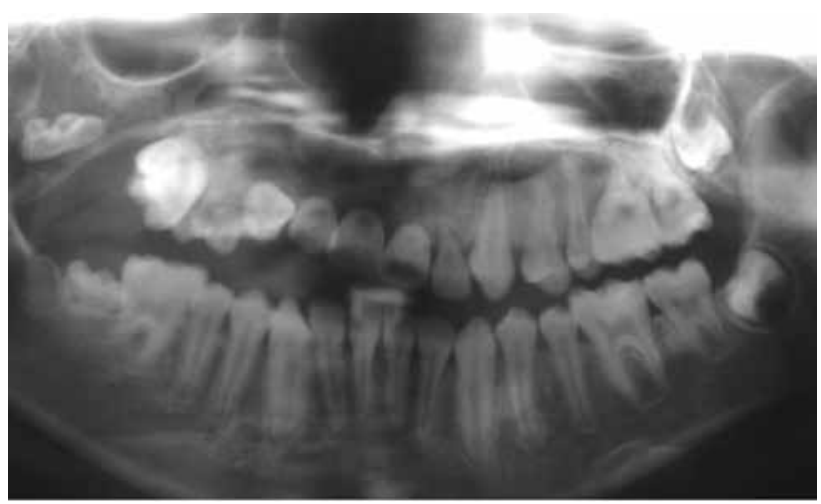

Figura 8. Radiografía panorámica.

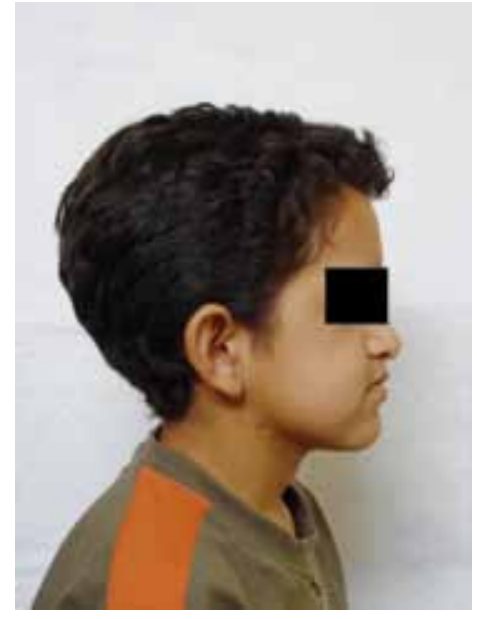

Figura 5. Vista lateral.

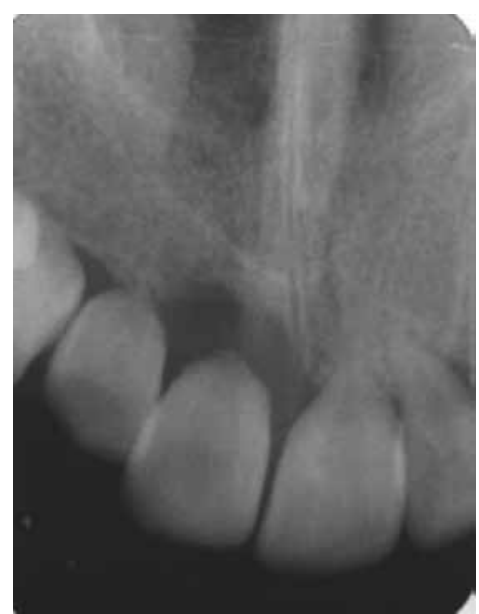

Figura 7. Radiografía anterosuperior.

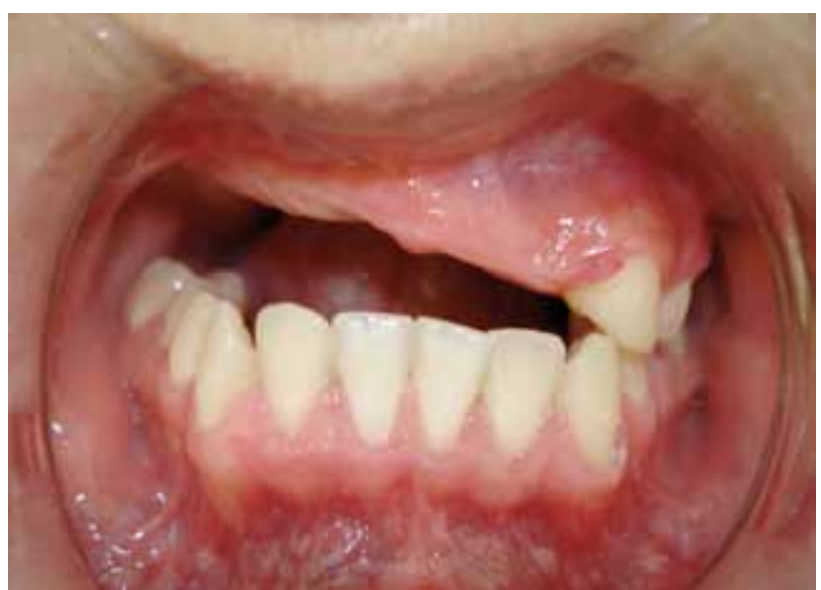

Figura 9. Vista intraoral final. Antes de colocación de prótesis. 
Dentro del plan de tratamiento, se realizan exodoncias de las piezas con amplia movilidad, y tratamiento periodontal, logrando dejar al paciente en condiciones optimas para utilizar una prótesis removible.

Caso Clínico 3: Paciente de sexo femenino de 9 años de edad con diagnóstico de Leucemia Linfática Aguda. Se encuentra hospitalizada iniciando terapia de inducción y cursa con neutropenia severa la cual genera mucositis IV y lesiones herpéticas en cavidad oral causándole gran dolor, limitación a la masticación y deglución.
Por lo que se empieza protocolo terapéutico con clorhexidina, analgésicos endovenosos y antimicóticos. Teniendo resultados a las 48 horas con mejoría notable.

Caso Clínico 4: Paciente de sexo masculino con antecedente de Linfoma No Hodgkin, $1^{\circ}$ en maxilar superior a los 9 años de edad. Dentro del esquema de tratamiento recibió quimioterapia y radioterapia. Sin recidiva de enfermedad.

Llega a consulta después de 12 años de haber completado su tratamiento, presentando asi-

\section{Caso Clínico 3}

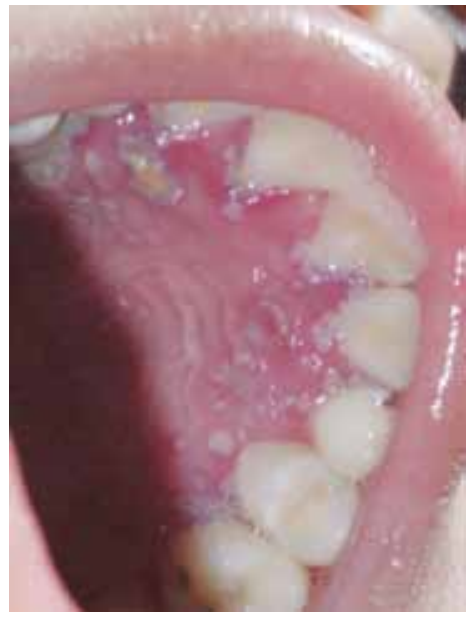

Figura 10. Lesiones vesiculares y tejido necrótico a nivel de mucosa de paladar duro.

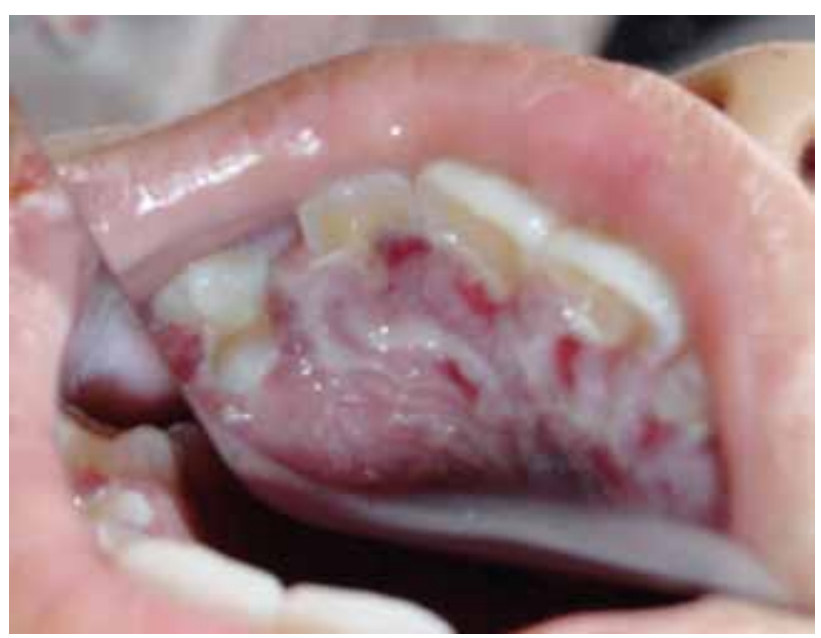

Figura 12. Lesiones ulcerosas y zonas necróticas a nivel de mucosa de paladar duro. Cursa con mucositis IV.

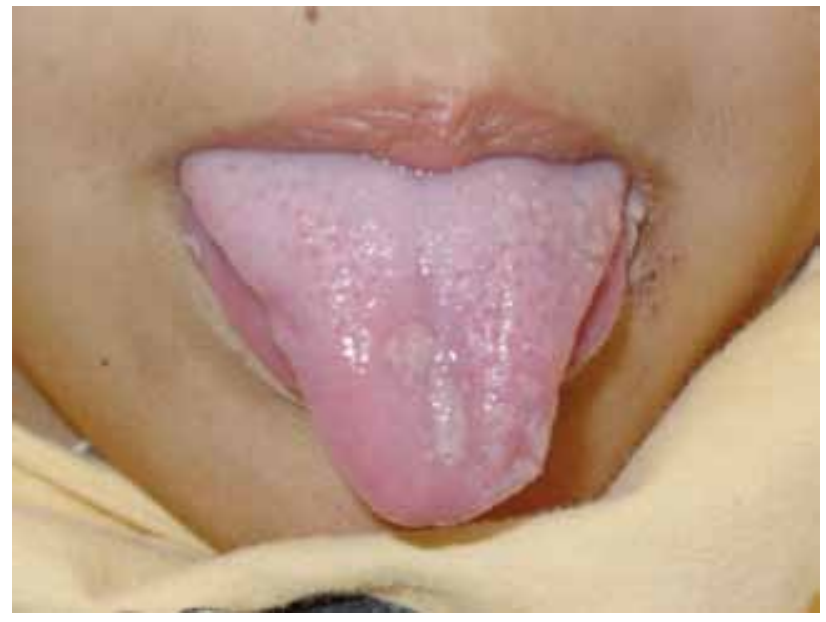

Figura 11. Lesiones herpéticas en labio inferior y en punta de lengua.

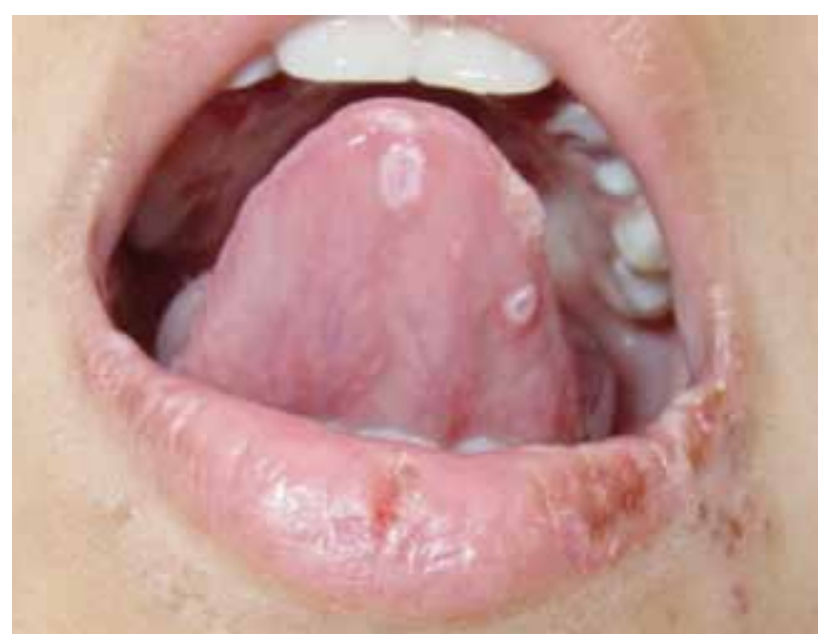

Figura 13. Lesiones vesiculares en dorso de lengua con lesiones herpéticas en labio inferior. 


\section{Caso Clínico 4}

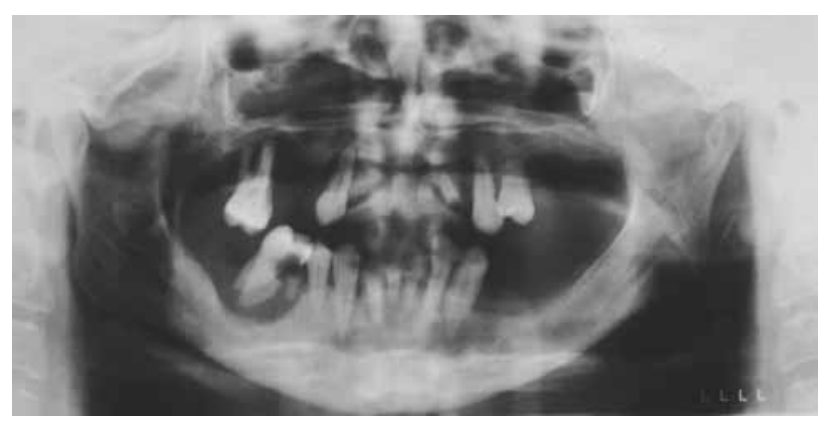

Figura 14. Radiografía Panorámica post tratamiento de radioterapia. Se evidencia alteración del proceso estilohioideo bilateral. Opacificación parcial de ambos senos maxilares. Rebordes óseos severamente reabsorbidos con zonas de abscesos periodontales. Procesos osteolíticos perirradiculares que comprometen la totalidad del soporte óseos dando aspecto de dientes flotantes. Edéntulo parcial ambos maxilares.

metría facial, hipoplasia maxilar, y múltiples lesiones cariosas. En la radiografía panorámica se observa calcificación segmentada del proceso estilohioideo bilateral. Opacificación parcial de ambos senos maxilares. Rebordes óseos severamente reabsorbidos con zonas de abscesos periodontales. Procesos osteolíticos perirradiculares que comprometen la totalidad del soporte óseos dando aspecto de dientes flotantes. Edéntulo parcial ambos maxilares.
Debido a la situación odontológica del paciente, sólo se podrá realizar tratamientos radicales de exodoncias y la preparación de la cavidad oral para la colocación de prótesis en ambos maxilares.

\section{Conclusiones}

Es importante conocer las lesiones características y complicaciones de las terapias antineoplásicas, diagnosticarlas y tratarlas para mejorar las condiciones de los pacientes.

Los esquemas preventivos son de gran ayuda, disminuyen la agresividad de los efectos tóxicos que se generan en las mucosas.

En los últimos años la radioterapia craneal ha sido reducida salvo en los casos estrictamente necesarios. Es importante conocer los riesgos que se generan durante las etapas de crecimiento y desarrollo dentocraneofacial.

Se recomienda individualizar el tratamiento de cada paciente. Los protocolos preestablecidos son de gran ayuda para una mejor atención hospitalaria.

\section{Bibliografía}

1. Cabrizo Merino MC, Oñate Sanchez RE. Aspectos odontoestomatológicos en oncología infantil. Med Oral Patol Oral Cir Bucal 2005; 10: 41-7.

2. Complicaciones orales del cáncer y de su terapia. www.med.uni-bonn.de/cancernet/302904.html Setiembre 2002.

3. Childers NK, Stinnett EA, Wheeler P. Oral complications in children with cancer. Oral Surg Oral Med Oral Pathol 1993; 75: 41-7.

4. Dos Santos Oliveira J, Ventiades JA, Fontana NN. Conducta odontológica em pacientes pediátricos portadores de leucemia.

5. Sepulveda E, Brethauer U, Jimenez M. Detección del virus herpes simple en lesiones de la mucosa oral en pacientes con terapia oncológica. Med Oral 2003; 8: 329-333.

6. Quasso L, Scipioni C, Pavesi L. Complicaciones periodontales en las leucemias en edad pediátrica. Av Periodon Implantol 2005; 17(2): 55-68. 
7. Pereira L, Batista L, Gordón -Nuñez MA. Prevention of oral lesions in children with acute lymphoblastic leukemia. International Journal of Pediatric Otorhinolaryngology 2006; 70: 1847-51.

8. Collard MM, Hunter ML. Dental care in acute lymphoblastic leukaemia: experiences of children and attitudes of parents. International Journal of Paediatric Dentistry 2001; 11: 274-80.

9. Nguyen AM. Manejo dental de pacientes que recibieron quimioterapia y radioterapia. AGD 1992-1993; 5: 47-56.

10. Meraw SJ, Reeve CM. Dental considerations and treatment of the oncology patient receiving radiation therapy. JADA 1998; 129: 201-205.

11. Sabater MM, Rodríguez ME, Muñoz J. Tratamiento de la mucositis oral con un protector de la mucosa. Dentum 2006; 6(1): 36-41.

12. Melo de Brito EM, Fernandes MZ, Bezerra L. Evaluation of an oral preventive protocol in children with acute lymphoblastic leukemia. Pesqui Odontol Bras 2003; 17(2): 147-50.

13. El-Housseiny AA, Saleh SM, El-Masry AA. Assessment of Oral Complications in Children receiving chemotherapy. J Clin Pediatr Dent 2007; 31(4): 267-73.

14. Castell PA, Basté MA, Viles MC. Prevención y tratamiento de La mucositis en El paciente onco-hematológico. Farmacia Hosp 2001; 25(3): 27-37.

15. Sabater MM, Lopéz J, Rodriguez de Rivera ME. Estado buco-dental y mucositis oral. Estudio clínico en pacientes con enfermedades hematológicas. Med Oral Patol Oral Cir Bucal 2006; 11: E497-502.

16. Gonzales H, Gonzales E, Zambrano O. Oral Candidiasis in children and adolescents with cáncer. Identification of Candida spp. Med Oral Patol Oral Cir Bucal 2007; 12(6): E419-423.

17. Alberth M, Majoros L, Kovalecz G. Significance of Oral Candida Infections in Children with Cancer. Pathology \& Oncology Research 2006; 12(4): 237-241.

18. Sepulveda E, Brethauer U, Rojas J. Ulceras orales en niños sometidos a quimioterapia: características clinicas y su relación con presencia de Virus Herpes Simple tipo I y Candida Albicans. Oral Med \& Pathol 2005; 10: E1-8.

19. Cheng KKF, Chamg AM, Yuen MP. Prevention of oral mucositis in paediatric patients treated with chemotherapy: a randomized crossover trial comparing two protocols of oral care. European Journal of Cancer 2004; 40: 1208-1216.

20. O’Sullivan E, Duggal MC, Bailey C. Changes in the oral microflora during cytotoxic chemoteraphy in children being treated for acute leukemia. International Journal of Paediatric Dentistry 1994; 4: 31-34.

21. Scully C, Sonis S. Oral mucositis. Oral Diseases 2006; 12: 229-41.

22. Cordova JC, Galvao C, Lunardi A. Oral Health and Dental Anormalies in patients treated for leukemia in childhood and adolescence. Peadiatr Blood Cancer 2009; 53: 361-65.

23. Miller AB. Reporting results of cancer treatment. Journal of the American Cancer Society 47: 207-214, 1981.

24. Vogler B, Ernsr E. Aloe Vera: a systemic review of its clinical effectiveness. British Journal of General Practice 1999; 49: 823-28.

25. Pecere T, Gazzola V, Mucignat C. Aloe-emodin is a new type of anticancer agent with selective activity against neuroectodermal tumors. Cancer research 2000; 60(1): 2800-4.

Recibido: 06-11-2009

Aceptado: 22-01- 2010

Correspondencia:sgru03@hotmail.com 